\title{
GABAergic synapse properties may explain genetic variation in hippocampal network oscillations in mice
}

\author{
Tim S. Heistek' ${ }^{1}$ A. Jaap Timmerman', Sabine Spijker ${ }^{2}$, Arjen B. Brussaard ${ }^{1}$ and Huibert D. Mansvelder ${ }^{*}$ \\ Department of Integrative Neurophysiology, Center for Neurogenomics and Cognitive Research, Neuroscience Campus Amsterdam, VU University, Amsterdam, \\ Netherlands \\ 2 Department of Molecular and Cellular Neuroscience, Center for Neurogenomics and Cognitive Research, Neuroscience Campus Amsterdam, VU University, \\ Amsterdam, Netherlands
}

\section{Edited by:}

Yehezkel Ben-Ari, Institut National de la Santé et de la Recherche Médicale,

France

\section{Reviewed by:}

Andreas Frick, Institut National de la Santé et de la Recherche Médicale,

France

Igor Timofeev, Laval University, Canada

*Correspondence:

Huibert D. Mansvelder, Department of Integrative Neurophysiology, Faculty of Earth and Life Sciences, De Boelelaan 1085, 1081 HV Amsterdam,

Netherlands.

e-mail:huibert.mansvelder@cncr.vu.nl
Cognitive ability and the properties of brain oscillation are highly heritable in humans. Genetic variation underlying oscillatory activity might give rise to differences in cognition and behavior. How genetic diversity translates into altered properties of oscillations and synchronization of neuronal activity is unknown. To address this issue, we investigated cellular and synaptic mechanisms of hippocampal fast network oscillations in eight genetically distinct inbred mouse strains. The frequency of carbachol-induced oscillations differed substantially between mouse strains. Since GABAergic inhibition sets oscillation frequency, we studied the properties of inhibitory synaptic inputs (IPSCs) received by CA3 and CA1 pyramidal cells of three mouse strains that showed the highest, lowest and intermediate frequencies of oscillations. In CA3 pyramidal cells, the frequency of rhythmic IPSC input showed the same strain differences as the frequency of field oscillations. Furthermore, IPSC decay times in both CA1 and CA3 pyramidal cells were faster in mouse strains with higher oscillation frequencies than in mouse strains with lower oscillation frequency, suggesting that differences in $\mathrm{GABA}_{\mathrm{A}}$-receptor subunit composition exist between these strains. Indeed, gene expression of $\mathrm{GABA}_{A}$-receptor $\beta 2$ (Gabrb2) and $\beta 3$ (Gabrb2) subunits was higher in mouse strains with faster decay kinetics compared with mouse strains with slower decay kinetics. Hippocampal pyramidal neurons in mouse strains with higher oscillation frequencies and faster decay kinetics fired action potential at higher frequencies. These data indicate that differences in genetic background may result in different $G_{A B A_{A}}$-receptor subunit expression, which affects the rhythm of pyramidal neuron firing and fast network activity through GABA synapse kinetics.

Keywords: fast network oscillations, GABA synapses, heritability, hippocampus, GABA receptor subunits, C57, Balbc, NOD

\section{INTRODUCTION}

Variation in cognitive abilities in humans can for a large part be explained by genetic variation. More than $85 \%$ of the variation in cognitive ability is of genetic origin (Posthuma et al., 2001a,b). Twin studies have shown that in humans properties of brain oscillations are also highly heritable (Posthuma et al., 2001b; Smit et al., 2005; Linkenkaer-Hansen et al., 2007). Brain oscillations are thought to be involved in cognition and different oscillation frequencies have been related to specific cognitive processes (Gray et al., 1989; Lopes da Silva, 1991; Llinas and Ribary, 1993). For instance, beta- and gamma-band oscillations are increased during working memory and selective attention tasks (Roelfsema et al., 1997; Tallon-Baudry et al., 1998, 2001; Fries et al., 2001; Womelsdorf et al., 2007). Findings in laboratory animals show that gamma-band oscillations in the hippocampus are most likely involved in memory encoding and retrieval (Lisman and Idiart, 1995; Montgomery and Buzsaki, 2007). Phase coupling of gamma oscillations between CA1 and CA3 increases during memory retrieval (Montgomery and Buzsaki, 2007), which occurs at different gamma frequencies than between CA1 and the enthorinal cortex (Colgin et al., 2009). In mice, hippocampal oscillations are affected by genetic background (Jansen et al., 2009). Genetic variation resulting in differences in brain oscillations may give rise to differences in cognition and intelligence in general. However, how genetic differences translate into differences in properties of oscillations and synchronization of neuronal activity is poorly understood.

To address this issue, we studied cellular and synaptic mechanisms underlying hippocampal fast network oscillations in acute brain slices of different mouse strains with known genetic differences. Muscarinic acetylcholine receptor (mAChR) activation in cortical and hippocampal networks acute brain slices induces synchronized activity and network oscillations. During these oscillations, synchronized activity of pyramidal neurons alternates with synchronized activity of inhibitory interneurons (Hajos et al., 2004; Mann et al., 2005; van Aerde et al., 2009). Cellular mechanisms underlying $\mathrm{mAChR}$-induced synchronization depend on synaptic communication between neurons (Fisahn et al., 1998; van Aerde et al., 2008, 2009). Ordered pyramidal and interneuron cell firing and inhibitory synaptic feedback constitute the underlying mechanism underlying oscillating neuronal networks at beta/gamma 
frequencies in all cortical areas (Fisahn et al., 1998; Hajos et al., 2004; Mann and Paulsen, 2005; Mann et al., 2005; Oren et al., 2006). Fast network oscillations in hippocampal slices, which resemble gamma frequency oscillations in hippocampus in vivo (Csicsvari et al., 2003; Mann et al., 2005), are generated within the CA3 region and depend on fast inhibitory transmission within the stratum pyramidale of CA3 (Mann et al., 2005). Inbred mouse strains show substantial differences in gene expression within the hippocampus (Fernandes et al., 2004; Hovatta et al., 2005) and show clear heritable differences in hippocampal oscillation properties (Jansen et al., 2009). To address the mechanisms underlying genetic variation in network oscillations, we studied CA3 pyramidal neuronal activity and the synaptic inputs they receive in eight different mouse strains during carbachol-induced fast network oscillations. We found that differences in oscillation frequency between mouse strains may be explained by differences in GABAergic synapse properties and $\mathrm{GABA}_{\mathrm{A}}$-receptor subunit expression.

\section{MATERIALS AND METHODS TISSUE PREPARATION}

All experimental methods involving animals were approved by the animal welfare committee of our university, and in accordance with Dutch and European law. The eight inbred mouse strains (129S1SvImJ, A/J, Balb/cByJ (Balbc), C3H/HeJ, C57Bl/6J (C57), $\mathrm{DBA} / 2 \mathrm{~J}, \mathrm{FVB} / \mathrm{NJ}$ and NOD/LtJ (NOD); Figure $1 \mathrm{~A}$ ) were obtained from Jackson Laboratories ${ }^{1}$. All animals were between 13- and 17-days old. After decapitation without anesthesia, brains were quickly removed and stored in ice cold artificial cerebrospinal fluid (ACSF) containing $125 \mathrm{mM} \mathrm{NaCl}, 25 \mathrm{mM} \mathrm{NaHCO}_{3}, 3 \mathrm{mM}$ $\mathrm{KCl}, 1.2 \mathrm{mM} \mathrm{NaH} \mathrm{PO}_{4}, 1 \mathrm{mM} \mathrm{CaCl}, 3 \mathrm{mM} \mathrm{MgSO}_{4}$, and $10 \mathrm{mM}$ $\mathrm{D}(+)$-glucose (carboxygenated with $5 \% \mathrm{CO}_{2} / 95 \% \mathrm{O}_{2}$ ). For RNA isolation, the ventral hippocampus was dissected on ice, and stored at $-80^{\circ} \mathrm{C}$ until further use. For electrophysiology, horizontal slices (400- $\mu \mathrm{m}$ thick $))$ from the ventral hippocampus were cut using a microtome $\left(\mathrm{Microm}^{2}\right)$. After preparation, slices were stored for at least $1 \mathrm{~h}$ in ACSF containing $2 \mathrm{mM} \mathrm{CaCl}_{2}$ and $2 \mathrm{mM} \mathrm{MgSO}_{4}$. To record field potentials, slices were placed on planar eight by eight multi-electrode arrays with $150 \mu \mathrm{m}$ spacing (MED-P5155, Panasonic ${ }^{3}$ ) or $200 \mu \mathrm{m}$ (Multichannel Systems ${ }^{4}$ ), which were coated with polyethylene (Sigma). Slices were left to properly attach to the electrodes for at least $1 \mathrm{~h}$ in a chamber with humidified carbogen gas before they were placed in the recording unit.

\section{ELECTROPHYSIOLOGY}

Local field potentials were measured in four slices simultaneous with four recording units at the same time using the multichannel system or in combination with patch-clamp recordings in the MED64 system. During recording slices were perfused with ACSF at a flow rate between 4 and $5 \mathrm{ml} / \mathrm{min}$ and were kept at $30^{\circ} \mathrm{C}$. Carbamoylcholine chloride (Carbachol, Sigma ${ }^{5}$ ) was perfused for at least $45 \mathrm{~min}$ or added after the mounting of the slices onto

\footnotetext{
${ }^{1}$ www.jax.com

${ }^{2}$ www.microm-online.com

${ }^{3}$ www.med64.com

${ }^{4}$ www.multichannelsystems.com

${ }^{5}$ www.sigmaaldrich.com
}

the multi-electrode grids. Spontaneous field potentials from all 64 recording electrodes were acquired simultaneously at $20 \mathrm{kHz}$ (MED64 system) or with 60 electrodes at $1 \mathrm{kHz}$ (multichannels systems). In addition to fast network oscillations we also observed low frequency network bursts in some slices of Balbc mice ( 9 out of 19) which occurred at a frequency of $0.54 \pm 0.11 \mathrm{~Hz}$.

Pyramidal cells in CA3 and CA1 neurons were recorded in whole-cell mode using a Multiclamp 700B amplifier (molecular devices ${ }^{6}$ ). Borosilicate glass (Harvard Apparatus ${ }^{7}$ ) electrodes with tip resistances of 2-5 MOhm were filled with intracellular solution containing $140 \mathrm{mM} \mathrm{K}$-gluconate, $1 \mathrm{mM} \mathrm{KCl}, 10 \mathrm{mM}$ HEPES, 4 mM K-phosphocreatine, 4 mM ATP-Mg, and 0.4 mM GTP (pH adjusted to 7.2 with $\mathrm{KOH}$ ). To ensure temporal alignment between the multi-electrode and single-cell electrophysiological signals, the current or voltage signals from the patch-clamp amplifiers were recorded on one channel of the MED64 amplifier, using an interface (Panasonic). IPSCs were recorded at $+20-\mathrm{mV}$ holding potential, while EPSCs were recorded at $-70 \mathrm{mV}$. Internal chloride concentrations were such that at these potentials IPSCs and EPSCs would have a different polarity. As the holding potential might influence the decay time kinetics (Collingridge et al., 1984) mIPSCs were recorded at $-70 \mathrm{mV}$ in presence of $25 \mu \mathrm{M}$ carbachol, $10 \mu \mathrm{M}$ 6,7-dinitroquinoxaline-2,3(1H, 4H)-dione (DNQX) and $20 \mu \mathrm{M}$ DL-AP5 and $1 \mu \mathrm{M}$ TTX and with use of intracellular solution with high chloride concentrations containing: $70 \mathrm{mM}$ Cs-gluconate, $70 \mathrm{mM} \mathrm{CsCl}, 10 \mathrm{mM}$ HEPES, $5 \mathrm{mM}$ ATP-Mg, and $0.5 \mathrm{mM}$ GTP and $5 \mathrm{mM}$ EGTA (pH adjusted to 7.2 with $\mathrm{CsOH}$ ). Zolpidem was obtained from Duchefa ${ }^{8}$.

\section{RNA ISOLATION, cDNA SYNTHESIS AND REAL-TIME QUANTITATIVE PCR (qPCR)}

After isolation of total RNA (Trizol, Invitrogen, San Diego, USA) according to standard procedures, a DNase-I treatment (2 $\mathrm{U}$, Invitrogen Carlsbad, CA, USA) to remove traces of genomic DNA was performed. The amount and purity of RNA was measured using a spectrophotometer (NanoDrop ND-1000 UV-Vis Spectrophotometer, Nanodrop Technologies ${ }^{9}$ ), and the integrity was analyzed by gel electrophoresis.

For mouse genes encoding $\mathrm{GABA}_{\mathrm{A}}$-receptor subunits, transcript-specific primers (Table 1) were designed based on Genbank sequence entries using Primer Express software (PE Biosystems ${ }^{10}$; manufacturer's settings). Only primers were taken of which endpoint PCRs showed the amplicon and no primer-dimers as determined by generation of dissociation curves, and which had high amplification efficiencies.

From each tissue sample ( $n=4$ per strain), random primed (hexamers; Eurogentec $\left.{ }^{11}\right)$ cDNA ( $1 \mu \mathrm{g}$ total RNA) was made with reverse transcriptase (200 U; Promega ${ }^{12}$; manufacturer's protocol). cDNA Aliquots were stored at $-80^{\circ} \mathrm{C}$, because repeated freeze-thaw

\footnotetext{
${ }^{6}$ www.moleculardevices.com

${ }^{7}$ www.harvardapparatus.com

${ }^{8}$ www.duchefa.com

${ }^{9}$ www.nanodrop.com

${ }^{10}$ www.appliedbiosystems.com

${ }^{11}$ www.eurogentec.com

${ }^{12}$ Www.promega.com
} 
Table 1 | Gene specific primers to different GABA ${ }_{A}$-receptor subunits and housekeeping genes used for real-time qPCR.

\begin{tabular}{|c|c|c|}
\hline Gene & Forward primer $5^{\prime}-\mathbf{3}^{\prime}$ & Reverse primer $5^{\prime}-3^{\prime}$ \\
\hline Gabra1 & GTCCTCTGCACCGAGAATTGC & TCAAATTCTTTAGGACAGAGGCAGTA \\
\hline Gabra2 & TGGTTTTTGCTTTGTACAGTCTGACT & GCAAATGCAGGTCTCCTTTAGAG \\
\hline Gabra4 & GCTCACTTAGCTTCCAGTCCAAA & GATGAAAGACCTCTGGCTGCA \\
\hline Gabra5 & AAAAGACATACAACAGCA & GAAAGTGCCAAACAAGATGGG \\
\hline Gabrb2 & СССАССTCCGGGAAACTC & GAAGACAAAGCACCCCATTAGG \\
\hline Gabrb3 & AAAGGATCGAGCTCCCACAGT & TGTGGCGAAGACAACATTCC \\
\hline Gabrg1 & TGCTTCGATAGACTGCAGAACTG & GAGTCAATTTTTGCAATGCGTATG \\
\hline Gabrg2 & TCCAAAAAGGCTGATGCTCACT & ACTCGACCATCATTCCAAATTCTC \\
\hline Gabrg3 & GAGGCCGCATCCACATTG & CAGGAAGGATGTCGGGAAGA \\
\hline
\end{tabular}

cycles affect measured cycle of threshold $(\mathrm{Ct})$ values. For qPCR measurements (ABI PRISM 7900, Applied Biosystems), PCR conditions $\left(50^{\circ} \mathrm{C}, 2 \mathrm{~min} ; 95^{\circ} \mathrm{C}, 10 \mathrm{~min} ; 40\right.$ cycles: $95^{\circ} \mathrm{C}, 10 \mathrm{~s}, 60^{\circ} \mathrm{C}$, $1 \mathrm{~min}$; dissociation curve in $10 \mathrm{~min}$ ) and SYBR green reagents (Applied Biosystems) were used in a reaction volume of $10 \mu \mathrm{l}$ using transcript-specific primers $(300 \mathrm{nM})$ on cDNA ( $\sim 5 \mathrm{ng}$ RNA equivalent).

The obtained Cycle of threshold value for every gene $\left(C t_{\mathrm{x}}\right)$ was used to calculate the relative level of gene expression by normalization to the geometric means of replicated reference controls $\left(\mathrm{Ct}_{\mathrm{HK}}\right.$; Gapdh, Hprt). GAPDH and HPRT were shown as stable expressing genes across strains (Loos et al., 2009). For all graphical representation and statistical calculations, $\log _{2}$-based values were used for amount of normalized transcript, $C-\left(C t_{\mathrm{x}}-C t_{\mathrm{HK}}\right)(C=5)$.

\section{DATA ANALYSIS}

Synaptic events were detected using Mini Analysis Program ${ }^{13}$. All other analysis was done using custom made scripts in igorPro 6.04 (Wavematrics $^{14}$ ) and matlab R2006B (The Mathworks ${ }^{15}$ ). Data was down sampled to $200 \mathrm{~Hz}-2 \mathrm{kHz}$ for analysis. Frequency and power of oscillations were calculated by fitting a Gaussian function to the Fourier transformed data. The average power and frequency of the oscillations were calculated separately in the different layers of the dentate gyrus, CA3 region and CA1 region by taking the average of all electrodes in that region. Synaptic events were selected by a threshold for amplitude and area which were equal across strains. IPSC $\tau$ decay times were calculated by fitting an exponential function to the individual IPSCs and only single synaptic events were used for fitting. The $\tau$ decay, amplitudes and interval times of a single neuron were put in a histogram and fitted with a lognormal for $\tau$ decay and amplitudes, or Gaussian distribution for interval times. The average IPSC/EPSC frequency was calculated as 1 /average interval time. Phase coupling factor was

\footnotetext{
${ }^{13}$ www.synaptosoft.com

${ }^{14}$ Www.wavematrics.com

${ }^{15}$ www.mathworks.com
}

calculated by wavelet transforming the local fied potential (LFP) of the electrode closest to the site of the whole-cell recording to determine the phase of the oscillations. Statistical analysis used either the Student's $t$ test (paired or unpaired) or an ANOVA with Student Newman Keuls post hoc test, as appropriate. For circular statistics we used Hotelling's test for paired comparisons and the Watson Williams test. The Rayleigh test was used to test whether the events showed significant phase-locking to the oscillation. Data are represented as average \pm SEM.

\section{RESULTS}

Recently, we reported that in eight genetically different inbred mouse strains variation in properties of fast hippocampal network oscillations can be partly explained by genetic differences (Jansen et al., 2009). To test whether these genetic differences are reflected in properties of excitatory and inhibitory synapses in CA 3 and CA1, which underlie fast network oscillations in these areas (Whittington et al., 1995; Mann et al., 2005) and information transfer between these areas, we recorded synaptic activity in CA1 and CA3 pyramidal neurons during fast network oscillations. Application of the mAChR agonist carbachol $(25 \mu \mathrm{M})$ to horizontal slices of the hippocampus induced fast network oscillations (Figure 1). The frequency ranged from 13.0 to $30.2 \mathrm{~Hz}$ in different mouse strains $(p<0.05$, ANOVA, Figures 1A-C) and post hoc analysis revealed a difference in frequency with maximal oscillation power (peak frequency) between Balbc $(17.5 \pm 0.8 \mathrm{~Hz}, n=13)$ and NOD mice $(21.6 \pm 1.2 \mathrm{~Hz}, n=10)(p<0.05$ Figure 1E). As was reported by Jansen et al. (2009), the power of oscillations in specific frequency bands differed between mouse strains (not shown). However, the power at the frequency that showed maximal power (peak power) in CA3 in each mouse strain was not different for the eight inbred strains ( $p=0.43$, ANOVA, Figure 1E).

Fast network oscillations in hippocampus are driven by rhythmic hyperpolarizing GABAergic inputs to CA3 pyramidal cells (Mann et al., 2005). To test whether properties of phasic GABAergic inputs to pyramidal cells can affect the frequency of oscillations, we recorded IPSCs in CA3 pyramidal neurons and slowed GABAergic 

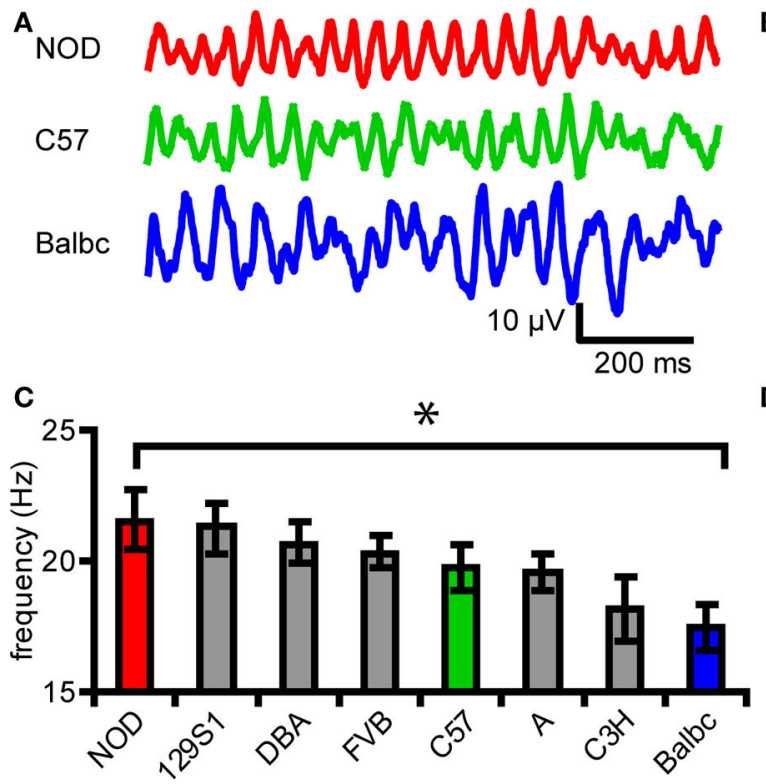

FIGURE 1 | Eight inbred mouse strains differ in frequency of fast network oscilliliations. (A) Example traces of field potential in CA3stratum radiatum from 3 different mouse strains (NOD, C57, Balbc). (B) Power spectral density
B
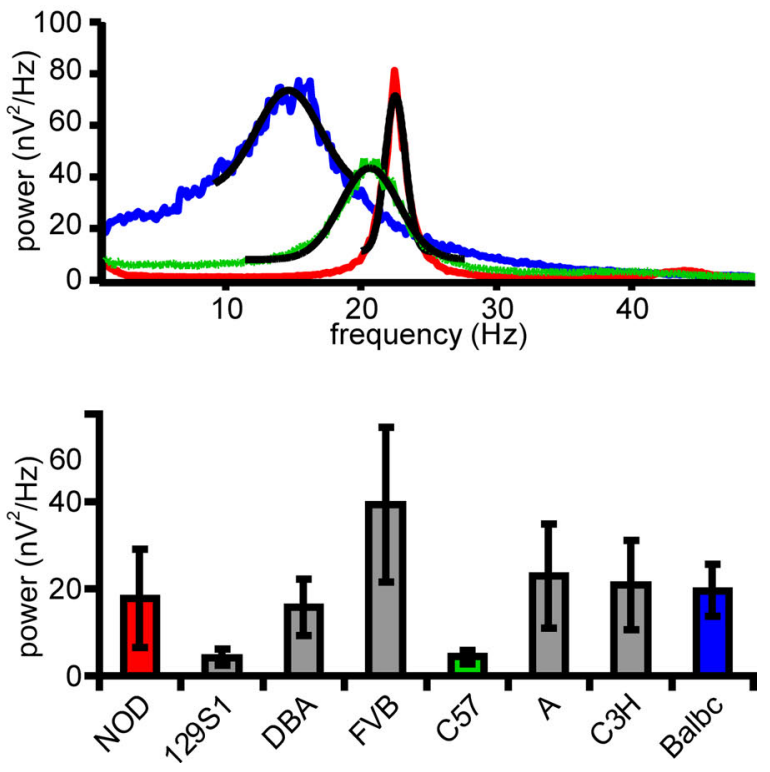

plot corresponding to the traces shown in B. Black lines show the Gaussian fits. (C) Peak frequency of oscillations in 8 different mouse strains. (D) Peak power of oscillations in 8 different mouse strains. Error bars show SEM. synaptic kinetics by applying the $\mathrm{GABA}_{\mathrm{A}}$-receptor modulator zolpidem during ongoing oscillations (Figure 2). Zolpidem $(1 \mu \mathrm{M})$ reduced the frequency of fast network oscillations in all mouse strains tested (to $82.0 \pm 1.8 \%$ of control; Figures $2 \mathrm{~A}-\mathrm{D}$ ). It also strongly increased the power of oscillations (to $234 \pm 35 \%$ ) $(p<0.05, n=9$, Figures 2A-D). These changes in frequency and power were similar in the whole hippocampus (Figure $2 \mathrm{C}$ ). At the synaptic level, zolpidem altered the properties of phasic GABAergic inhibition in NOD and Balbc mice (Figures 2E-H; zolpidem data grouped for both strains). Zolpidem decreased the IPSC frequency (to $76 \pm 2.5 \%$ of control), and increased the decay time constant $(178 \pm 10 \%)$ and IPSC amplitude $(175 \pm 20 \%)(p<0.05, n=9$, Figures $2 \mathrm{E}-\mathrm{H})$. We also observed an increased phase-locking of IPSCs to the field potential ( $163 \pm 29 \%$, Figures 2 F,G) and a shift in the average IPSC phase relative to the field from $1.04 \pm 0.17$ radials to $0.17 \pm 0.30$ radials ( $p<0.05$, Figures $2 \mathrm{~F}, \mathbf{H})$. Thus, decreasing IPSC frequency and increasing IPSC decay time constant with zolpidem decreased the frequency of fast network oscillations. The increase in IPSC amplitude and the increase in the power of oscillations may be explained by more precise timing of IPSC inputs in the presence of zolpidem, reflected by an enhanced IPSC phaselocking (Figure 2G).

Since GABAergic synaptic properties and IPSC kinetics can strongly affect the frequency of fast network oscillations, we investigated whether differences exist in GABAergic synaptic transmission received by CA3 pyramidal neurons of NOD and Balbc mice. In whole-cell recordings from CA3 pyramidal neurons we monitored both IPSCs (at $+20 \mathrm{mV}$ ) and EPSC (at $-70 \mathrm{mV}$ ) during fast network oscillations. The frequency of IPSCs received by CA3 pyramidal cells was significantly higher in NOD mice $(24.6 \pm 1.0 \mathrm{~Hz}, n=7)$ than in Balbc mice $(20.6 \pm 1.1 \mathrm{~Hz}$, $n=6$ ), while in C57 mice pyramidal neurons received IPSCs at intermediate frequencies $(21.7 \pm 0.9 \mathrm{~Hz}, n=8)(p<0.05$, Figures 3A-C). In each mouse strain, the IPSC frequency received by CA3 pyramidal neurons was very similar to the frequency of fast network oscillations in that particular strain (Figure 3E). In addition to higher frequencies of IPSCs, GABAergic inputs to CA3 pyramidal neurons in NOD mice also had a significantly faster $\tau$ decay time constant $(8.3 \pm 0.7 \mathrm{~ms})$ compared to Balbc mice $(10.7 \pm 0.4 \mathrm{~ms})(p<0.05$, Figures 3D,E), while IPSCs in C57 mice showed intermediate decay time constants $(9.0 \pm 0.7 \mathrm{~ms})$. Just as IPSC frequency, IPSC kinetics paralleled the frequency of oscillations in different mouse strains: NOD mice showed faster network oscillations and faster IPSC decay kinetics, whereas Balbc mice showed slower network oscillations and slower IPSC decay kinetics. IPSC amplitude, phase-locking and IPSC timing relative to the field were not different between different mouse strains $(p>0.05$, Figures 3F-I), in line with the absence of difference between power of fast network oscillations between NOD and Balbc mice (Figure 1E). These data suggest that differences in kinetics of GABAergic inhibition received by CA3 pyramidal neurons may explain the differences in fast network oscillations in genetically distinct mouse strains.

We next tested whether differences exist in excitatory glutamatergic transmission received by CA3 pyramidal neurons in NOD and Balbc mice. Spontaneous EPSCs recorded in CA3 pyramidal cells of NOD mice arrived at a similar frequency as in Balbc mice and were timed similarly related to the network oscillations (Figures 4A-C,E,F). However, the amplitude of excitatory inputs were larger in Balbc mice ( $57.5 \pm 6.5 \mathrm{pA}, n=6)$ than in NOD mice $(39.1 \pm 2.7 \mathrm{pA}, n=7$; $p<0.05$, Figures $4 \mathrm{D}, \mathrm{E})$. Thus, lower frequencies of phasic GABAergic inhibition and fast network oscillations in Balbc mice are not reflected by an altered frequency of excitatory transmission, but by increased amplitudes of EPSCs. 


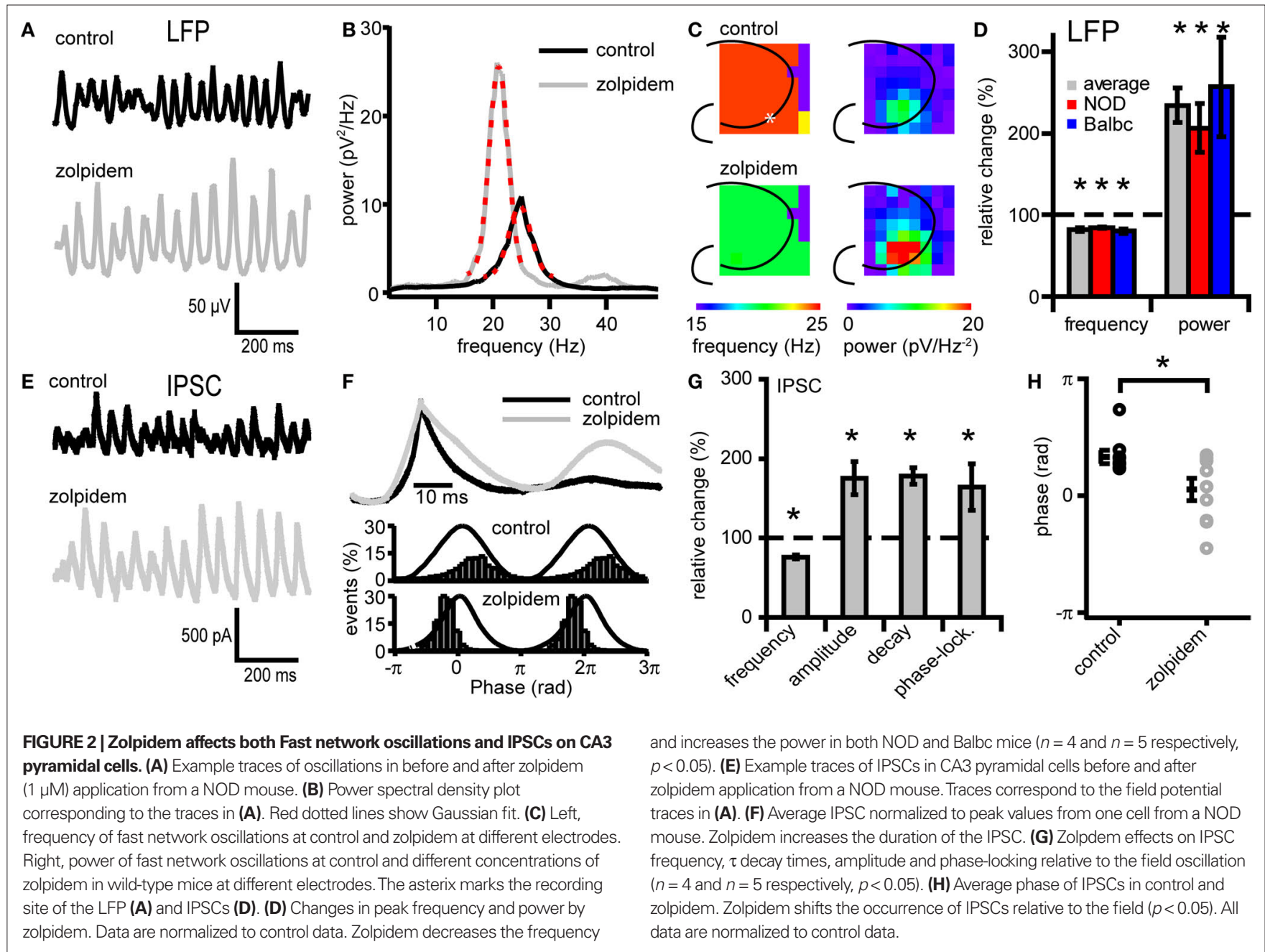

Carbachol-induced fast network oscillations in the CA1 area of the hippocampus are driven by rhythmic activity of CA3 circuits (Fisahn et al., 1998; Mann et al., 2005). Physically dissociating CA1 from CA3 in hippocampal slices silences carbachol-induced oscillations in CA1, but not in CA3 (Fisahn et al., 1998). Within a mouse strain, carbachol-induced fast network oscillations in CA1 had the same frequency as oscillations in CA3. Between mouse strains, oscillation frequency in CA1 showed similar differences as was the case for CA3 (NOD: $19.7 \pm 0.5 \mathrm{~Hz}$; Balbc: $16.9 \pm 0.6 \mathrm{~Hz}$, $p<0.05$, data not shown). To test whether spontaneous GABAergic inhibition received by CA1 pyramidal cells during ongoing oscillations showed similar differences between NOD and Balbc mice as inhibition received by CA3 pyramidal cells, we recorded IPSCs in CA1 pyramidal neurons. The IPSC inputs to CA1 pyramidal cells were less timed than in $\mathrm{CA} 3$ and showed lower phase-locking $r$ values $(p<0.05$, Figures 3G,H, and 5D). Although the IPSC decay kinetics were faster in CA1 compared with CA3 pyramidal cells, we observed a similar difference in decay time constant between NOD $(6.4 \pm 0.3 \mathrm{~ms}, n=11)$ and Balbc $(8.8 \pm 0.6 \mathrm{pA}$, $n=7)(p<0.05$, Figures $5 \mathbf{A}, \mathbf{B})$. We observed no differences in IPSC frequency between NOD and Balbc ( $p>0.05$, Figure 5C), neither did we find differences in IPSC amplitude, phase-locking or phase (Figure 5D, average data not shown).
Altered decay kinetics of GABAergic synaptic activity most likely results from differences in $\mathrm{GABA}_{\mathrm{A}}$-receptor subunit expression in GABAergic synapses (Brussaard et al., 1997; Brussaard and Herbison, 2000; Bosman et al., 2005). To test whether differences in $\mathrm{GABA}_{\mathrm{A}}$-receptor subunits exist in hippocampus of NOD and Balbc mice, we compared mRNA levels of different $\mathrm{GABA}_{\mathrm{A}}$-receptor subunits in hippocampus. We observed no differences in expression of $\alpha$ or $\gamma$ subunits, however there was a significant higher expression of the $\beta 2$ and $\beta 3$ subunits in NOD mice compared with Balbc mice ( $p=0.008$ and $p=0.020$, both $n=4$, Figure 6A), suggesting that GABAergic synapses in NOD mice may have a different molecular composition or stoichiometry of subunits.

To test whether differences in $\mathrm{GABA}_{\mathrm{A}}$-receptor subunit expression in hippocampus of NOD and Balbc mice was reflected in altered kinetics of single GABAergic synapses, we recorded miniature IPSCs (mIPSCs) from CA3 pyramidal neurons while preventing action potential firing by blocking sodium channels with TTX. In the presence of Carbachol, decay time constants of mIPSCs in NOD mice were faster than in Balbc mice (NOD: $6.8 \pm 0.3 \mathrm{~ms}$, $n=5$; Balbc: $7.8 \pm 0.2 \mathrm{~ms}, n=8$; $p<0.05$, Figures 6B,C). The frequency of mIPSCs did not differ between the mouse strains (Figure 6D). IPSCs from CA1 pyramidal cells showed a similar difference in decay time constants (data not shown). These data 


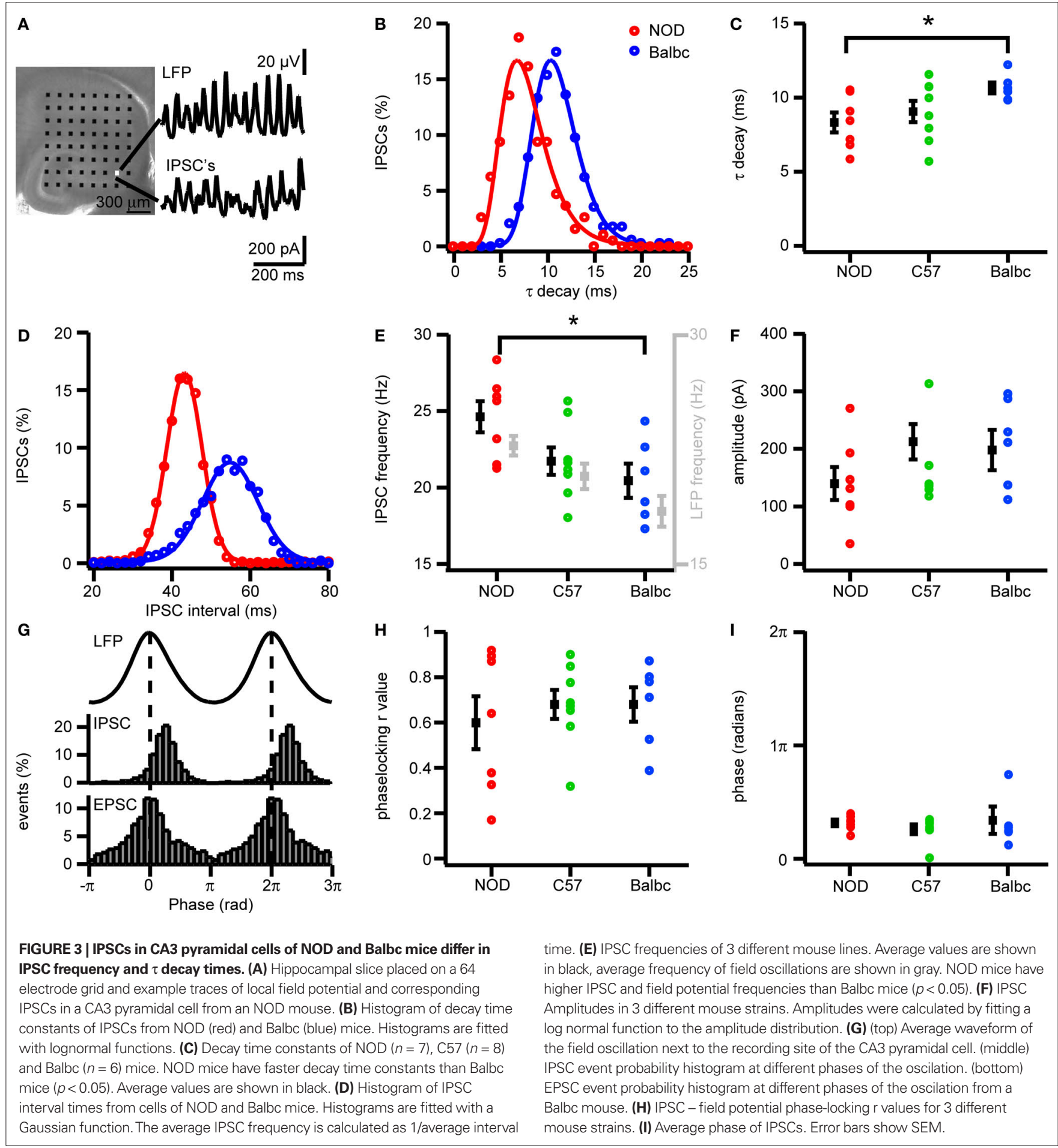

show that GABAergic synapses on hippocampal pyramidal neurons show different kinetics that most likely result from differences in $\mathrm{GABA}_{\mathrm{A}}$-receptor subunit expression. These differences in subunit expression may explain the higher frequency of fast network oscillations in hippocampus of NOD mice compared with Balbc mice.

The pyramidal cell-interneuron feedback model for fast network oscillations predicts that the spike timing and spiking probability of pyramidal cells depends on the decay time of inhibitory inputs
(Traub et al., 2000). Since decay times of IPSCs differed between NOD and Balbc mice, we tested whether hippocampal pyramidal cells showed differences in action potential firing during fast network oscillations in these mouse strains. In cell-attached recordings during carbachol-induced oscillations, the AP firing frequency of CA3 pyramidal cells was significantly higher in NOD $(6.1 \pm 1.0 \mathrm{~Hz})$ compared to Balbc mice $(3.7 \pm 0.6 \mathrm{~Hz}, p<0.05$, Figures 7A,B). All CA3 cells showed significant phase-locking to the field oscillations 


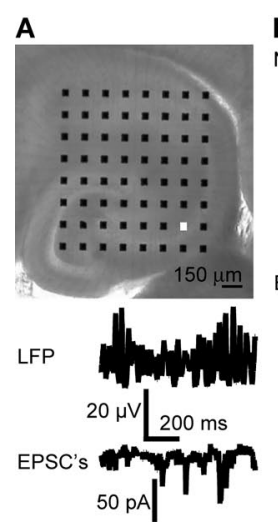

B

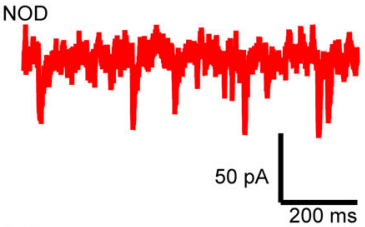

Balbc

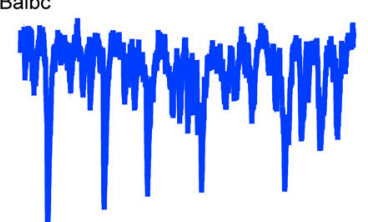

FIGURE 4 | EPSCs in CA3 pyramidal cells in NOD and Balbc mice differ in amplitude. (A) Hippocampal slice placed on a 64 electrode grid and example traces of local field potential and corresponding EPSCs in a CA3 pyramidal cell from a NOD mouse recorded at the in the white colored electrode in the picture. (B) Example
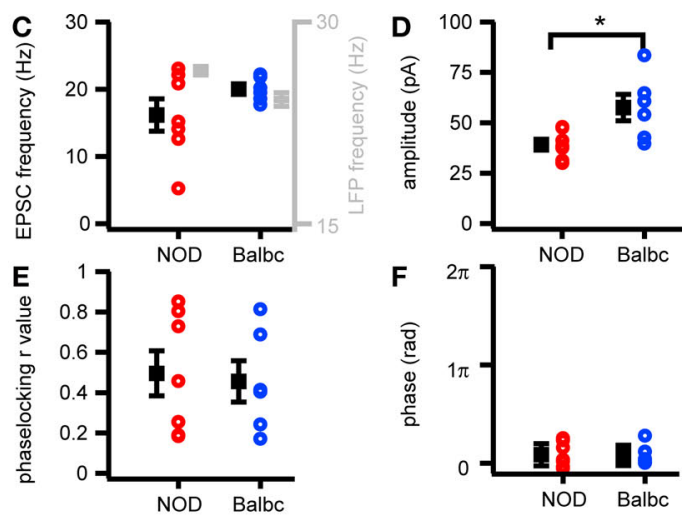

traces of EPSCs in NOD and Balbc mice. (C) EPSC frequency in NOD $(n=7)$ and Balbc $(n=6)$ mice. (D) Average EPSC amplitude is higher in Balbc mice compared to NOD mice $(p<0.05)$. (E) IPSC - field potential phase-locking $r$ values for NOD and Balbc mice. (F) Average phase of IPSCs. Average values + SEM are shown in black.

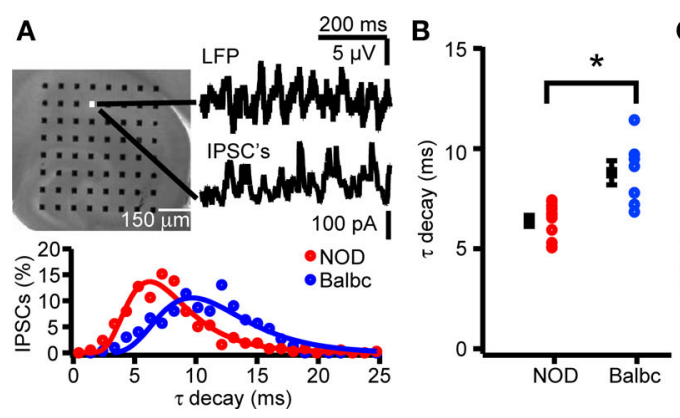

FIGURE 5 | IPSCs in CA1 pyramidal cells of NOD and Balb mice differ in $\tau$ decay times. (A) Hippocampal slice (top) placed on a 64 electrode grid and example traces of local field potential and corresponding IPSCs in a CA1 pyramidal cell from an NOD mouse. Histogram (bottom) of decay time constants of IPSCs from NOD and Balbc mice. Histograms are fitted with lognormal functions. (B) Decay time constants of NOD $(n=11)$ and Balbc $(n=7)$
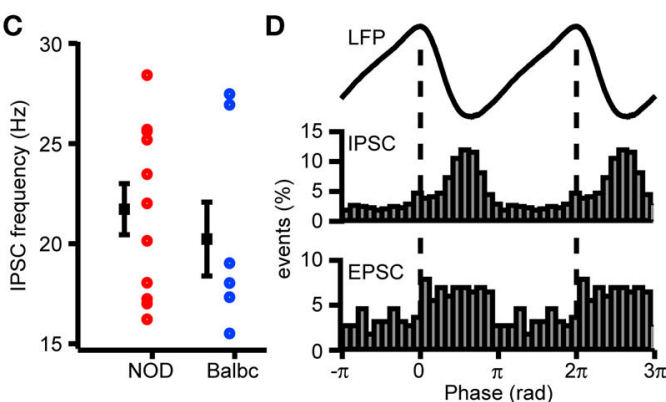

mice. NOD mice have faster decay time constants than Balbc mice $(p<0.05)$. (C) IPSC frequencies of NOD and Balbc mice. (D) (top) Average waveform of the field oscillation next to the recording site of the CA1 pyramidal cell. (middle) IPSC event probability histogram at different phases of the oscilation. (bottom) EPSC event probability histogram at different phases of the oscilation from an NOD mouse. Average values + SEM are shown in black.
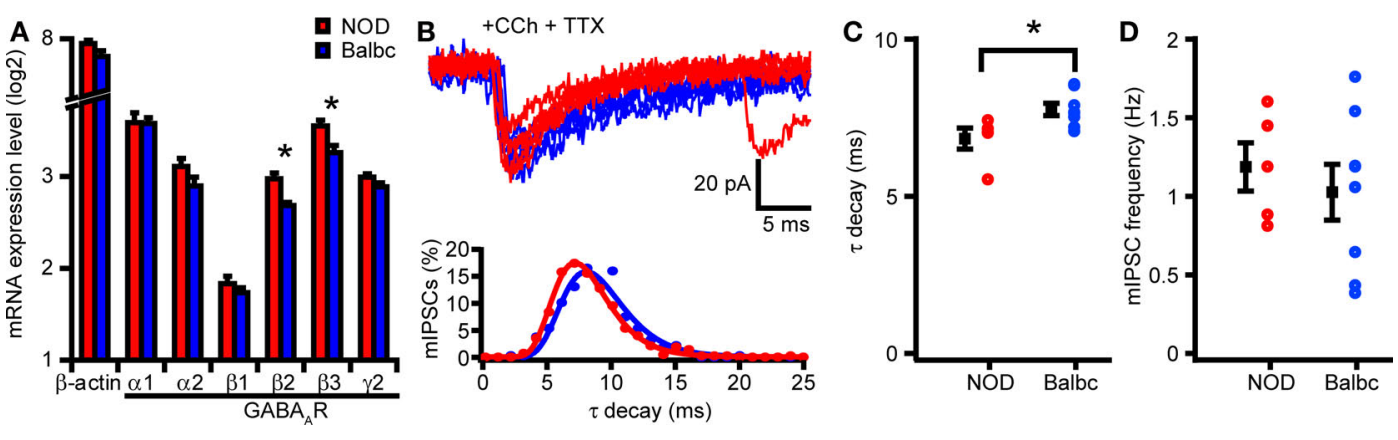

FIGURE 6 | NOD and Balbc mice strains differ in mRNA expression of GABA $A_{A}$-receptor subunits $\boldsymbol{\beta} 2$ and $\boldsymbol{\beta} 3$ and mIPSC kinetics. (A) mRNA expression levels of $\beta$ actin (Actb) and different $G A B A_{A}$-receptor subunits in NOD and Balbc mice in the hippocampus. There is a significant difference in mRNA expression of the GABA $A_{A}$-receptor subunits $\beta 2$ (Gabrb2) and $\beta 3$ (Gabrb3) between NOD and Balbc mice (both $n=4, p<0.05$ ). (B) Example traces of
mIPSCs from CA3 pyramidal cells from NOD and Balbc mice (top) and histogram (bottom) of decay time constants of IPSCs from NOD and Balbc mice. Histograms are fitted with lognormal functions. (C) Decay time constants of $\operatorname{NOD}(n=5)$ and Balbc $(n=8)$ mice. NOD mice have faster decay time constants then Balbc mice $(p<0.05)$. (D) Average frequencies of mIPSCs of NOD and Balbc mice. Average values + SEM are shown in black. 

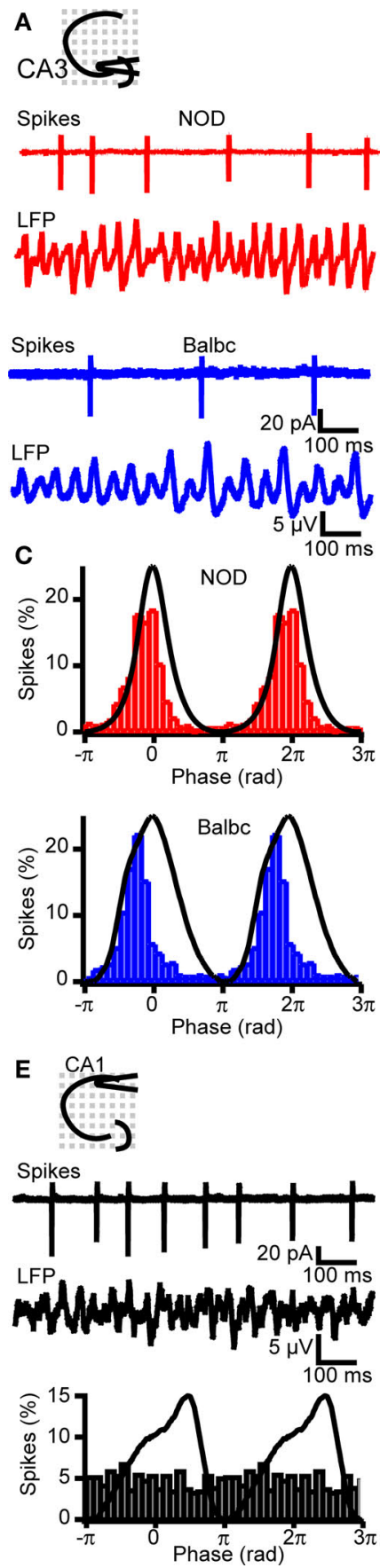

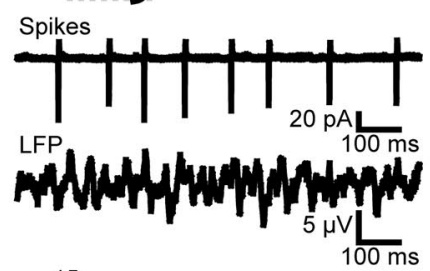

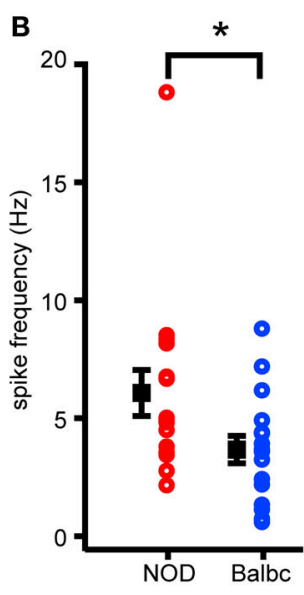

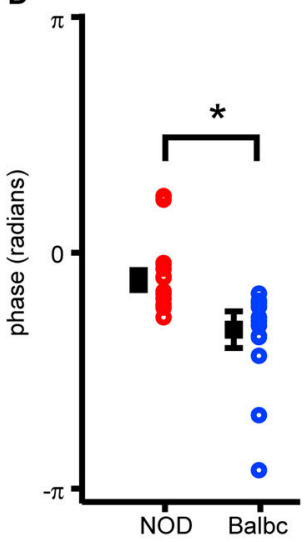

$\mathbf{F}$

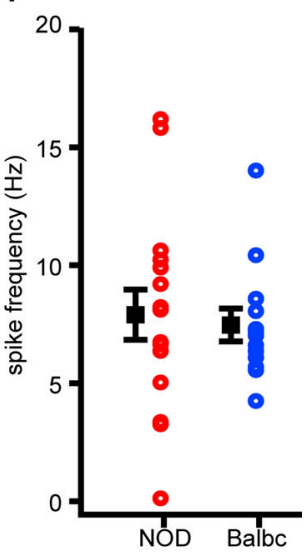

FIGURE 7 | Spike timing and frequency differs between NOD and Balbc mice. (A) Example traces of spikes recorded from CA3 pyramidal cells in cell-attached mode and field potential in NOD and Balbc mice. (B) Average firing frequency of CA3 pyramidal cells in NOD $(n=16)$ is higher than in Balbc ( $n=16, p<0.05$ ) mice. (C) Average waveform of the field oscillation spike probability histogram at different phases of the oscilation from a cell from a NOD (top) and a Balbc (bottom) mouse. (D) I. Average phase of spikes. Spikes in CA3 pyramidal cells from Balbc mice occur at a earlier phase than in NOD mice $(p<0.05)$. (E) Example traces of spikes recorded from CA1 pyramidal cells from a Balbc mouse in cell-attached mode and field potential (top) and average spike probability histogram at different phases of the oscillation (bottom). (F) Average firing frequency of CA1 pyramidal cells in NOD and Balbc mice. Average values + SEM are shown in black.
(Figure 7C) in both mouse strains. However, the timing of spiking occurred around the peak of the oscillation in pyramidal neurons of NOD mice $(-0.05 \pm 0.02 \pi)$, while in Balbc mice most spikes occurred before the peak $(-0.16 \pm 0.04 \pi, p<0.05$, Figures 7C,D). Unlike CA3 pyramidal cells only 4 out of 16 CA1 pyramidal cells in NOD mice and 2 out of 13 CA1 pyramidal cells in Balbc mice fired phase-locked to the oscillations in CA1 (Figure 7E). Furthermore, there was no difference in firing frequency between NOD and Balbc mice in CA1 pyramidal cells (Figure 7F). These data indicate that the decay kinetics of GABAergic synapses may affect the timing and frequency of CA3 pyramidal cell firing as well as the frequency of fast network oscillations.

\section{DISCUSSION}

Brain oscillations are thought to be involved in cognition (Gray et al., 1989; Lopes da Silva, 1991; Llinas and Ribary, 1993), and properties of brain oscillations are also highly heritable (Deary et al., 2010). Little is known about the mechanisms by which genetic variation affects synchronized neuronal activity. In this study, we investigated what mechanisms may give rise to differences in fast network oscillations in hippocampus of genetically distinct inbred mouse strains. Our main findings are that: (1) Genetically distinct mouse lines show differences in the frequency of fast network oscillations that are paralleled by similar differences in the frequency of inhibitory inputs received by CA3 pyramidal neurons. (2) Differences in frequency of oscillations are also paralleled by differences in GABAergic synapse decay kinetics in CA3 and CA1 pyramidal cells. (3) In mouse strains with the highest frequency of oscillations and fastest GABA synapse kinetics, there is more gene expression of $G_{A B A}$-receptor $\beta 2$ and $\beta 3$ subunit mRNA. (4) In NOD mice, which show the fastest field oscillations, CA3 pyramidal cells fire at a higher frequency and at a later phase of the field oscillations than in Balbc mice. Our findings suggest that differences in genetic background result in different GABA -receptor subunit expression. This may affect GABA synapse kinetics and thereby action potential firing of CA3 pyramidal cells and the frequency of fast network oscillations.

GABAergic inhibition is critical for fast network oscillations, both in hippocampus and neocortex (Whittington et al., 1995; Fisahn et al., 1998; Traub et al., 2000; Mann et al., 2005; Atallah and Scanziani, 2009; van Aerde et al., 2009). Recurrent feedback loops between pyramidal cells and inhibitory interneurons underlie cholinergically-induced gamma oscillations (Fisahn et al., 1998; Traub et al., 2000; Mann et al., 2005). In the CA3 region of the hippocampus, peri-somatic inhibition of CA3 pyramidal neurons appears to dictate the time window during which these neurons fire action potentials (Hajos et al., 2004; Mann et al., 2005). Rapid adjustments in inhibition can instantaneously modulate oscillation frequency (Atallah and Scanziani, 2009). We find that the frequency of inhibitory inputs received by CA3 pyramidal neurons is similar to the frequency of field oscillations. In addition, in genetically different mouse strains the inhibitory input frequency differs to a similar extent as the field oscillations. Therefore, one may predict that the window during which the CA3 pyramidal neuron can escape inhibition and fire an action potential may be shorter in NOD mice due to higher IPSC frequencies. 
However, this appears not be the case, since mouse strains that showed higher IPSC frequencies, also showed faster IPSC decay kinetics. In line with other studies (Fisahn et al., 1998; Cope et al., 2005), we found that the modulation of IPSC decay kinetics directly affects the frequency of oscillations. Decay kinetics of peri-somatic inhibition in pyramidal neurons most likely also determines the time window during which the cell can fire action potentials. In cells that receive IPSCs with faster decay kinetics, the episode of inhibition during which the threshold for spiking is elevated will most likely be shorter. Thereby, the combination of IPSCs that arrive more frequent but with faster decay kinetics entrains action potential firing of CA3 pyramidal neurons at higher frequencies. Thus, faster GABAergic synapses may give rise to faster recurrent feedback loops between pyramidal cells and interneurons. Thereby, NOD mice may experience faster field oscillations than Balbc mice. Allosteric enhancement of GABA decay kinetics not only reduced the frequency, but also increased the power of oscillations. In addition to decay times of IPSCs, zolpidem also affected IPSC amplitudes. Since hippocampal field potential oscillations are a reflection of inhibitory synaptic potentials (Oren et al., 2010), this may explain the increase in oscillation power caused by zolpidem. Since we did not observe a different in IPSC amplitude between the mouse strains, this may explain the absence of a difference in oscillation peak power between mouse strains.

Both inhibitory and excitatory synaptic transmission in CA3 are required for rhythm generation. It was shown recently that excitation and inhibition fluctuate in balance during kainate-induced oscillations (Atallah and Scanziani, 2009). However, in carbachol-induced oscillations the phase-locking of excitatory input was much smaller then the phase-locking of inhibitory input, which is in agreement with earlier studies showing that pyramidal cells receive phase-locked inhibition while interneurons receive phase locked excitation during carbachol-induced oscillations (Oren et al., 2006). The amplitude of EPSCs was larger in the mouse strain that showed the slowest oscillation frequency, Balbc. It is unclear what the underlying cause for larger amplitude EPSCs in these mice is. It was recently described that field oscillations are predominantly shaped by inhibitory inputs received by CA3 pyramidal cells (Oren et al., 2010). Therefore, the difference in EPSC amplitude between mouse strains was most likely not reflected in the frequency of field oscillations.

$\mathrm{GABA}_{\mathrm{A}}$-receptor subunit distribution in hippocampal synapses has been described to some extend in the CA1 region (Fritschy and Brunig, 2003), however receptor subunit distribution in perisomatic inhibitory synapses in CA3 is not known. We found that NOD mice showed faster IPSC kinetics and had higher mRNA levels of both $\beta 2$ as well as $\beta 3$ subunits. GABA $\alpha$ and $\beta$ subunits affect decay time kinetics ((Brussaard et al., 1997; Brussaard and

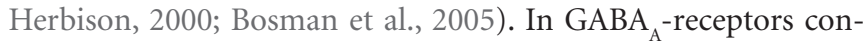
taining $\alpha 5$ and $\gamma 2$ subunits, and $\beta 2$ and $\beta 3$ subunits affect GABA currents in opposite directions: receptors with $\beta 2$ subunits had the highest desensitization rate, while receptors with $\beta 3$ showed the lowest desensitization rate (Burgard et al., 1996). How increased expression of both $\beta 2$ and $\beta 3$ subunits affects GABA receptor kinetics is not clear at this point. Also, it is not known how $\beta$ subunits

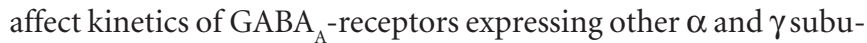
nits than $\alpha 5$ and $\gamma 2$. In transgenic mice lacking $\beta 3$ subunits, decay time kinetics of IPSCs in cortical and hippocampal neurons are faster (Ramadan et al., 2003; Hentschke et al., 2009), and gamma oscillations in the hippocampus are smaller and faster (Hentschke et al., 2009). However in these mice not only the $\beta 3$ subunit is lacking, but also the expression of $\alpha 2$ and $\alpha 3$ subunits is reduced, which may affect decay time kinetics (Ramadan et al., 2003). This may suggest that $\beta$ subunits could affect protein levels of other $\alpha$ subunits, thereby affecting receptor kinetics.

IPSC kinetics in CA1 pyramidal neurons was faster than in CA3 pyramidal neurons. Fritschy and Brunig (2003) showed that in CA1 pyramidal neurons peri-somatic inhibitory synapses predominantly contain $\alpha 1$ subunits. We have evidence that inhibitory inputs to CA3 pyramidal neurons may be mainly mediated by $\alpha 2$ containing receptors (Tim S. Heistek and Huibert D. Mansvelder, unpublished observations), which could explain the differences in IPSC kinetics between these regions. Whether regional differences in $\beta 2$ and $\beta 3$ subunit expression exist in hippocampus is not known.

Although it is likely that GABAergic synapse kinetics can account for the observed variation in oscillation frequency between mouse lines, there might be other genes that contribute to differences in properties of fast network oscillations in these strains. While manipulation of ionic channels affecting the excitability of pyramidal cells, such as Im, Ih and Icat (Fisahn et al., 2002; Leao et al., 2009), mainly affects the power of hippocampal oscillations, manipulation of interneuron excitability does affect the frequency of oscillations (Mann and Mody, 2010). Whether differences in interneuron excitability exist between NOD and Balbc mice is not known and Balbc mice show differences in behavior, including anxiety and impulsivity behavior (Fernandes et al., 2004; Hovatta et al., 2005; Loos et al., 2009). It might very well be that genes involved in anxiety and impulsivity also influence neuronal oscillatory activity. Behavioral studies showed that spatial learning and hippocampal LTP are affected in some of these mouse strains (Nguyen et al., 2000; Nguyen and Gerlai, 2002). In recombinant inbred mouse lines there is a high variability in protein kinase C (PKC) levels which correlates with spatial learning (Wehner et al.,

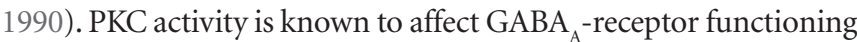
and trafficking (Song and Messing, 2005) and could thereby also contribute to the variation in frequency of oscillations.

\section{GENETICS AND HERITABILITY OF OSCILLATIONS}

Differences in properties of oscillations might explain heritable variations in cognitive ability. Cognitive functions require the integrated activity of multiple specialized, distributed brain areas. Phase coupling between different areas of the hippocampus and cortex is thought to regulate the information flow between brain areas (Canolty et al., 2006; Sirota et al., 2008; Colgin et al., 2009). Small differences in frequency of oscillations in the hippocampus may lead to a different coupling efficiency and thereby influence spatial and temporal memory processes. Therefore it is likely that genetic variation influencing GABAergic inhibition could impact on neuronal network dynamics and cognition. Indeed in humans there are indications that polymorphisms in $\mathrm{GABA}_{\mathrm{A}}$-receptor properties are related with beta- and gamma-band power of neuronal oscillations (Porjesz et al., 2002). Furthermore, in schizophrenia patients there is a decrease in gamma-band activity (Cho et al., 2006) that is correlated with increased expression of the $\mathrm{GABA}_{\mathrm{A}}$-receptor $\alpha 2$ subunit and a decrease in GABA transporters(Lewis et al., $2005,2008)$. However little is known about variation in frequency 
within the gamma band and its implication for cognition. As the frequency range of gamma oscillations in vivo is much broader than observed in hippocampal slices, it will be challenging to test whether variation in the frequency range of gamma oscillations exists between individuals.

\section{REFERENCES}

Atallah, B. V., and Scanziani, M. (2009). Instantaneous modulation of gamma oscillation frequency by balancing excitation with inhibition. Neuron 62, 566-577.

Bosman, L. W., Heinen, K., Spijker, S., and Brussaard, A. B. (2005). Mice lacking the major adult GABAA receptor subtype have normal number of synapses, but retain juvenile IPSC kinetics until adulthood. J. Neurophysiol. 94, 338-346.

Brussaard, A. B., and Herbison, A. E. (2000). Long-term plasticity of postsynaptic GABAA-receptor function in the adult brain: insights from the oxytocin neurone. Trends Neurosci. 23, 190-195.

Brussaard, A. B., Kits, K. S., Baker, R. E., Willems, W. P., Leyting-Vermeulen, J. W., Voorn, P., Smit, A. B., Bicknell, R. J., and Herbison, A. E. (1997). Plasticity in fast synaptic inhibition of adult oxytocin neurons caused by switch in GABA(A) receptor subunit expression. Neuron 19, 1103-1114.

Burgard, E. C., Tietz, E. I., Neelands, T. R., and Macdonald, R. L. (1996). Properties of recombinant gammaaminobutyric acid A receptor isoforms containing the alpha 5 subunit subtype. Mol. Pharmacol. 50, 119-127.

Canolty, R. T., Edwards, E., Dalal, S. S., Soltani, M., Nagarajan, S. S., Kirsch, H. E., Berger, M. S., Barbaro, N. M., and Knight, R. T. (2006). High gamma power is phase-locked to theta oscillations in human neocortex. Science 313, 1626-1628.

Cho, R. Y., Konecky, R. O., and Carter, C. S. (2006). Impairments in frontal cortical gamma synchrony and cognitive control in schizophrenia. Proc. Natl. Acad. Sci. U.S.A. 103, 19878-19883.

Colgin, L. L., Denninger, T., Fyhn, M., Hafting, T., Bonnevie, T., Jensen, O., Moser, M. B., and Moser, E. I. (2009). Frequency of gamma oscillations routes flow of information in the hippocampus. Nature 462, 353-357.

Collingridge, G. L., Gage, P. W., and Robertson, B. (1984). Inhibitory postsynaptic currents in rat hippocampal CA1 neurones. J. Physiol. (Lond.) 356, 551-564.

Cope, D. W., Halbsguth, C., Karayannis, T., Wulff, P., Ferraguti, F., Hoeger, H., Leppa, E., Linden, A. M., Oberto, A., Ogris, W., Korpi, E. R., Sieghart, W., Somogyi, P., Wisden, W., and Capogna,
M. (2005). Loss of zolpidem efficacy in the hippocampus of mice with the GABAA receptor gamma2 F77I point mutation. Eur. J. Neurosci. 21, 3002-3016.

Csicsvari, J., Jamieson, B., Wise, K. D., and Buzsaki, G. (2003). Mechanisms of gamma oscillations in the hippocampus of the behaving rat. Neuron 37 , 311-322.

Deary, I. J., Penke, L., and Johnson, W. (2010). The neuroscience of human intelligence differences. Nat. Rev. Neurosci. 11, 201-211.

Fernandes, C., Paya-Cano, J. L., Sluyter, F., D'Souza, U., Plomin, R., and Schalkwyk, L.C. (2004). Hippocampal gene expression profiling across eight mouse inbred strains: towards understanding the molecular basis for behaviour. Eur. J. Neurosci. 19, 2576-2582.

Fisahn, A., Pike, F. G., Buhl, E. H., and Paulsen, O. (1998). Cholinergic induction of network oscillations at $40 \mathrm{~Hz}$ in the hippocampus in vitro. Nature 394, 186-189.

Fisahn, A., Yamada, M., Duttaroy, A., Gan, J. W., Deng, C. X., McBain, C. J., and Wess, J. (2002). Muscarinic induction of hippocampal gamma oscillations requires coupling of the $\mathrm{M} 1$ receptor to two mixed cation currents. Neuron 33, 615-624.

Fries, P., Reynolds, J. H., Rorie, A. E., and Desimone, R. (2001). Modulation of oscillatory neuronal synchronization by selective visual attention. Science 291, 1560.

Fritschy, J. M., and Brunig, I. (2003). Formation and plasticity of GABAergic synapses: physiological mechanisms and pathophysiological implications. Pharmacol. Ther. 98, 299-323.

Gray, C. M., Konig, P., Engel, A. K., and Singer, W. (1989). Oscillatory responses in cat visual cortex exhibit inter-columnar synchronization which reflects global stimulus properties. Nature 338, 334-337.

Hajos, N., Palhalmi, J., Mann, E. O., Nemeth, B., Paulsen, O., and Freund, T. F. (2004). Spike timing of distinct types of GABAergic interneuron during hippocampal gamma oscillations in vitro. J. Neurosci. 24, 9127-9137.

Hentschke, H., Benkwitz, C., Banks, M. I., Perkins, M. G., Homanics, G. E., and Pearce, R. A. (2009). Altered GABAA,slow inhibition and network oscillations in mice lacking the

\section{ACKNOWLEDGMENTS}

We thank Hans Lodder and Rik Jansen for excellent technical assistance. Funding for this work was provided by grants from NWO (917.76.360), VU University board (Stg VU-ERC) and Neurobsik (www.neurobsik.nl) to Huibert D. Mansvelder.

GABAA receptor beta3 subunit. J. Neurophysiol. 102, 3643-3655.

Hovatta, I., Tennant, R. S., Helton, R. Marr, R. A., Singer, O., Redwine, J. M., Ellison, J. A., Schadt, E. E., Verma, I. M., Lockhart, D. J., and Barlow, C. (2005). Glyoxalase 1 and glutathione reductase 1 regulate anxiety in mice. Nature 438, 662-666.

Jansen, R.,Linkenkaer-Hansen, K.,Heistek, T., Timmerman, J., Mansvelder, H. D., Brussaard, A. B., de Gunst, M., and van Ooyen, A. (2009). Inbred mouse strains differ in multiple hippocampal activity traits. Eur. J. Neurosci. 30, 1092-1100.

Leao, R. N., Tan, H. M., and Fisahn, A. (2009). Kv7/KCNQ channels control action potential phasing of pyramidal neurons during hippocampal gamma oscillations in vitro. J. Neurosci. 29, 13353-13364.

Lewis, D. A., Cho, R. Y., Carter, C. S. Eklund, K., Forster, S., Kelly, M. A., and Montrose,D. (2008). Subunit-selective modulation of GABA type A receptor neurotransmission and cognition in schizophrenia. Am. J. Psychiatry 165, 1585-1593.

Lewis, D. A., Hashimoto, T., and Volk, D. W. (2005). Cortical inhibitory neurons and schizophrenia. Nat. Rev. Neurosci. 6, 312-324.

Linkenkaer-Hansen, K., Smit, D. J., Barkil, A., van Beijsterveldt, T. E. Brussaard, A. B., Boomsma, D. I. van Ooyen, A., and de Geus, E. J. (2007). Genetic contributions to long-range temporal correlations in ongoing oscillations. J. Neurosci. 27, 13882-13889.

Lisman, J. E., and Idiart, M. A. (1995). Storage of $7 \pm 2$ short-term memories in oscillatory subcycles. Science 267, 1512-1515.

Llinas, R., and Ribary, U. (1993). Coherent 40-Hz oscillation characterizes dream state in humans. Proc. Natl. Acad. Sci. U.S.A. 90, 2078-2081.

Loos, M., van der Sluis, S., Bochdanovits, Z., van Zutphen, I. J., Pattij, T., Stiedl, O., Smit, A. B., and Spijker, S. (2009). Activity and impulsive action are controlled by different genetic and environmental factors. Genes Brain Behav. 8, 817-828

Lopes da Silva, F. (1991). Neural mechanisms underlying brain waves: from neural membranes to networks. Electroencephalogr. Clin. Neurophysiol. 79, 81-93.
Mann, E. O., and Mody, I. (2010). Control of hippocampal gamma oscillation frequency by tonic inhibition and excitation of interneurons. Nat. Neurosci. 13,205-212.

Mann, E. O., and Paulsen, O. (2005). Mechanisms underlying gamma ('40 Hz') network oscillations in the hippocampus-a mini-review. Prog. Biophys. Mol. Biol. 87, 67-76.

Mann, E. O., Suckling, J. M., Hajos, N., Greenfield, S. A., and Paulsen, O. (2005). Perisomatic feedback inhibition underlies cholinergically induced fast network oscillations in the rat hippocampus in vitro. Neuron 45 , 105-117.

Montgomery, S. M., and Buzsaki, G. (2007). Gamma oscillations dynamically couple hippocampal CA3 and CA1 regions during memory task performance. Proc. Natl. Acad. Sci. U.S.A. 104, 14495-14500.

Nguyen, P. V., Abel, T., Kandel, E. R., and Bourtchouladze, R. (2000). Straindependent differences in LTP and hippocampus-dependent memory in inbred mice. Learn. Mem. 7 , 170-179.

Nguyen, P. V., and Gerlai, R. (2002). Behavioural and physiological characterization of inbred mouse strains: prospects for elucidating the molecular mechanisms of mammalian learning and memory. Genes Brain Behav. $1,72-81$.

Oren, I., Hajos, N., and Paulsen, O. (2010). Identification of the current generator underlying cholinergically induced gamma frequency field potential oscillations in the hippocampal CA3 region. J. Physiol. 588, 785-797.

Oren, I., Mann, E. O., Paulsen, O., and Hajos, N. (2006). Synaptic currents in anatomically identified CA3 neurons during hippocampal gamma oscillations in vitro. J. Neurosci. 26, 9923-9934.

Porjesz, B., Almasy, L., Edenberg, H. J., Wang, K., Chorlian, D. B., Foroud, T., Goate, A., Rice, J. P., O'Connor, S. J., Rohrbaugh, J., Kuperman, S., Bauer, L. O., Crowe, R. R., Schuckit, M. A., Hesselbrock, V., Conneally, P. M., Tischfield, J.A., Li, T. K., Reich, T., and Begleiter, H. (2002). Linkage disequilibrium between the beta frequency of the human EEG and a GABAA receptor gene locus. Proc. Natl. Acad. Sci. U.S.A. 99, 3729-3733. 
Posthuma, D., de Geus, E. J., and Boomsma, D. I. (2001a). Perceptual speed and IQ are associated through common genetic factors. Behav. Genet. 31, 593-602.

Posthuma, D., Neale, M. C., Boomsma, D. I., and de Geus, E. J. (2001b). Are smarter brains running faster? Heritability of alpha peak frequency, IQ, and their interrelation. Behav. Genet. 31, 567-579.

Ramadan, E., Fu, Z., Losi, G., Homanics, G.E., Neale, J.H., and Vicini, S. (2003). GABA(A) receptor beta3 subunit deletion decreases alpha $2 / 3$ subunits and IPSC duration. J. Neurophysiol. 89, 128-134.

Roelfsema, P. R., Engel, A. K., König, P., and Singer, W. (1997). Visuomotor integration is associated with zero time-lag synchronization among cortical areas. Nature 385, 157-161.

Sirota, A., Montgomery, S., Fujisawa, S., Isomura, Y., Zugaro, M., and Buzsaki, G. (2008). Entrainment of neocortical neurons and gamma oscillations by the hippocampal theta rhythm. Neuron 60, 683-697.
Smit, D. J., Posthuma, D., Boomsma, D. I., and Geus, E. J. (2005). Heritability of background EEG across the power spectrum. Psychophysiology 42, 691-697.

Song, M., and Messing, R. O. (2005). Protein kinase $\mathrm{C}$ regulation of GABAA receptors. Cell. Mol. Life Sci. 62, 119-127.

Tallon-Baudry, C., Bertrand, O., and Fischer, C. (2001). Oscillatory synchrony between human extrastriate areas during visual short-term memory maintenance. J. Neurosci. 21, RC177.

Tallon-Baudry, C., Bertrand, O., Peronnet, F., and Pernier, J. (1998). Induced gamma-band activity during the delay of a visual short-term memory task in humans. J. Neurosci. 18, 4244-4254.

Traub, R. D., Bibbig, A., Fisahn, A., LeBeau, F. E., Whittington, M. A., and Buhl, E. H. (2000). A model of gamma-frequency network oscillations induced in the rat CA3 region by carbachol in vitro. Eur. J. Neurosci. 12, 4093-4106. van Aerde, K. I., Heistek, T. S., and Mansvelder, H. D. (2008). Prelimbic and infralimbic prefrontal cortex interact during fast network oscillations. PLoS ONE 3, e2725. doi: 10.1371/journal.pone.0002725.

van Aerde, K. I., Mann, E. O., Canto, C. B., Heistek, T. S., Linkenkaer-Hansen, K., Mulder, A. B., van der Roest, M., Paulsen, O., Brussaard, A. B., and Mansvelder, H. D. (2009). Flexible spike timing of layer 5 neurons during dynamic beta oscillation shifts in rat prefrontal cortex. J. Physiol. (Lond.) 587, 5177-5196.

Wehner, J. M., Sleight, S., and Upchurch, M. (1990). Hippocampal protein kinase $\mathrm{C}$ activity is reduced in poor spatial learners. Brain Res. 523, 181-187.

Whittington, M. A., Traub, R. D., and Jefferys, J. G. (1995). Synchronized oscillations in interneuron networks driven by metabotropic glutamate receptor activation. Nature 373 , 612-615.

Womelsdorf, T., Schoffelen, J. M., Oostenveld, R., Singer, W., Desimone, R., Engel, A. K., and Fries, P. (2007). Modulation of neuronal interactions through neuronal synchronization. Science 316, 1609.

Conflict of Interest Statement: The authors declare that the research was conducted in the absence of any commercial or financial relationships that could be construed as a potential conflict of interest.

Received: 23 February 2010; paper pending published: 10 March 2010; accepted: 06 May 2010; published online: 02 June 2010.

Citation: Heistek TS, Timmerman AJ, Spijker S, Brussaard AB and Mansvelder $H D$ (2010) GABAergic synapse properties may explain genetic variation in hippocampal network oscillations in mice. Front. Cell. Neurosci. 4:18. doi: 10.3389/ fncel.2010.00018

Copyright $\odot 2010$ Heistek, Timmerman, Spijker, Brussaard and Mansvelder. This is an open-access article subject to an exclusive license agreement between the authors and the Frontiers Research Foundation, which permits unrestricted use, distribution, and reproduction in any medium, provided the original authors and source are credited. 